\title{
ANÁLISE DA ABORDAGEM GAMMA-PHI NA MODELAGEM TERMODINÂMICA DE SISTEMAS BINÁRIOS CONTENDO COMPONENTES PRESENTES NO BIODIESEL
}

\section{L.P. BARREIROS, P.F. ARCE-CASTILLO}

\author{
Universidade de São Paulo, Escola de Engenharia de Lorena, Departamento de \\ Engenharia Química \\ E-mail para contato: leonardo.pb@alunos.eel.usp.br
}

\begin{abstract}
RESUMO - O presente trabalho visa realizar um estudo da modelagem do equilíbrio liquido-vapor (ELV) por meio da abordagem Gamma-Phi, apropriada para cálculos a baixas pressões, de sistemas binários de componentes presentes na mistura reacional do biodiesel obtido pela rota etílica. O modelo estudado é a equação cúbica de estado de Peng-Robinson (EdE PR), a qual descreve o comportamento da fase vapor, em termos dos coeficientes de fugacidade, e para descrever a fase liquida, em termos dos coeficientes de atividade, utilizouse o modelo de energia livre de Gibbs em excesso, Non-Randon Two Liquids (NRTL). Os resultados obtidos por meio da modelagem termodinâmica foram comparados com dados experimentais (obtidos da literatura) determinando assim se é possível aplicar a abordagem Gamma-Phi para predizer o ELV para sistemas binários submetidos a baixas pressões.
\end{abstract}

\section{INTRODUÇÃO}

O Brasil é um dos maiores consumidores de diesel do mundo. Devido à alta demanda interna desde 1982, fizeram-se estudos sobre a substituição de derivados do petróleo por combustíveis renováveis. As pesquisas foram feitas com óleos vegetais brutos e esteres obtidos pela rota etílica, embora os resultados tenham sido bons, a tecnologia não permitia a viabilização econômica da produção em larga escala. Atualmente as pesquisas estão avançando cada vez mais no sentido do desenvolvimento de biocombustíveis. Neste cenário, o biodiesel tem ganhado espaço por ser menos poluente que seu equivalente fóssil além de não emitir partículas de enxofre e outros componentes próprios dos derivados de petróleo. No Brasil é especialmente vantajoso investir em pesquisas nesta área, não só pelo país possuir a maior produção de etanol do mundo como também por suas grandes lavouras de soja, principal matéria prima fonte de ácidos graxos para a produção de biodiesel (Pacheco, 2006).

\subsection{Obtenção do Biodiesel pela Rota Etílica}

O biodiesel é um conjunto de ésteres de cadeia longa, gerados pela reação entre ácidos graxos e álcoois (metanol e etanol). Esta reação normalmente se processa com excesso de álcool e um catalizador básico. O produto deste processo é uma mistura bifásica contendo ésteres, água, glicerol além de álcool não reagido e catalizadores (Coelho, 2011). O metanol atualmente é o álcool mais utilizado para a produção de biodiesel, devido às vantagens econômicas como menores custos, tempo de reação, consumo de vapor e maior rendimento. 
No Brasil devido à grande oferta de etanol, abre-se a possibilidade de trabalhar pela rota etílica (menos toxico que o metanol).

\subsection{Recuperação de Subprodutos e Reagentes}

O glicerol é o principal subproduto da reação de transesterificação e assim como o biodiesel também possui valor agregado se for adequadamente separado, o álcool que entra em excesso também pode ser separado e retornar ao reator melhorando assim o rendimento do processo e o lucro de produção. Estas separações podem ser feitas a custos relativamente baixos e sob condições amenas, através do controle de variáveis como temperatura e pressão aliadas ao estudo da composição durante o equilíbrio termo-físico do sistema (Mendes et. al., 2012). A partir de modelos preditivos, baseados no conceito de energia livre de Gibbs, é possível realizar a modelagem termodinâmica do ELV utilizando a abordagem Gamma-Phi com o método de Bolha T ou Bolha P.

\subsection{Abordagem Gamma-Phi.}

Os modelos preditivos baseados na "idealidade" do equilíbrio de fases são a lei de Raoult e lei de Raoult modificada, que apesar de incluir o coeficiente de atividade, levando em conta as não idealidades da fase liquida, o modelo é fortemente dependente de considerações de idealidade para a fase vapor. A abordagem mais eficiente, para descrever o comportamento da fase vapor de uma mistura, é através dos coeficientes de fugacidade $\left(\widehat{\emptyset}_{i}^{V}\right)$, que é obtido pela EdE Peng-Robinson, e para descrever o comportamento da fase líquida, costuma-se utilizar os coeficientes de atividade $\left(\gamma_{i}\right)$ obtido através de um modelo de energia livre de Gibbs em excesso. Com esses dois critérios se verificar a condição de isofugacidade (Equação 1) (Smith et al., 2005).

$$
\hat{f}_{i(p, T, x i)}^{L}=\hat{f}_{i(p, T, y i)}^{V} \text { ou } x_{i} \gamma_{i} \emptyset_{i}^{S A T} P_{i}^{S A T} \exp \left[\frac{\underline{U}_{i}^{L}\left(p-p_{i}^{S A T}\right)}{R T}\right]=y_{i} \widehat{\emptyset}_{i}^{V} P
$$

\subsubsection{Non-Random Two Liquids (NRTL)}

É um dos modelos mais bem-sucedidos. Baseado na teoria dos dois fluidos juntamente com a teoria de composição local, é capaz de ser aplicado tanto a misturas homogêneas como a sistemas com miscibilidade parcial abrangendo tanto o equilíbrio líquido-líquido (ELL) quanto o equilíbrio líquido vapor (ELV). O modelo NRTL é constituído por três parâmetros ajustáveis e pode ser adaptado a sistemas multicomponentes e altamente não lineares (Terron, 2009). O modelo de energia livre de Gibbs em excesso, NRTL, foi desenvolvido por Renon e Prausnitz (Renon e Prausnitz, 1968) e pode ser expresso de acordo com as Equações 2 e 3.

$$
\begin{aligned}
& \frac{g^{E}}{R T}=x_{1} x_{2}\left[\frac{\tau_{21} G_{21}}{x_{1}+x_{2} G_{21}}+\frac{\tau_{12} G_{12}}{x_{2}+x_{1} G_{12}}\right] \\
& \tau_{12}=\frac{g_{12}-g_{22}}{R T} \quad, \quad \tau_{21}=\frac{\mathrm{g}_{21}-\mathrm{g}_{11}}{\mathrm{RT}} \quad, \quad G_{21}=\exp \left(-\alpha \tau_{21}\right)
\end{aligned}
$$


Os parâmetros ajustáveis são $\alpha, g_{12}$ e $g_{21}$; onde o parâmetro ' $\alpha$ ' é adimensional enquanto os parâmetros $g_{12}$ e $g_{21}$ encontram-se em unidades de energia por mol.

\subsubsection{EdE Peng-Robinson.}

Na década de 1970, foi proposta a EdE Peng-Robinson (PR) (Peng e Robinson, 1976), que ainda é muito utilizada na modelagem termodinâmica devido a sua eficiência e versatilidade, principalmente na predição do comportamento do equilíbrio de fases. Para predizer o comportamento de fases do ELV, a EdE PR precisa de propriedades críticas dos componentes do sistema e de parâmetros de interação binaria usados nas regras de mistura. A EdE PR é capaz de predizer propriedades termodinâmicas da mistura tanto na fase liquida quanto na fase vapor simultaneamente (Terron, 2009) e pode ser apresentada em termos do fator de compressibilidade (Equação 4).

$$
Z^{3}-(1-B) Z^{2}+\left(A-2 B-3 B^{2}\right) Z-A B+B^{2}+B^{3}=0
$$

Neste trabalho, são preditas as propriedades termodinâmicas: temperatura e pressão de bolha, por meio da abordagem assimétrica Gamma-Phi com a EdE PR e o modelo de energia livre de Gibbs em excesso, NRTL, para modelar termodinamicamente sistemas binários de compostos presentes na mistura reacional do biodiesel.

\section{MATERIAIS E METODOS}

Para o desenvolvimento do algoritmo de cálculo utilizou-se o software MATLAB, a EdE PR para o cálculo dos coeficientes de fugacidade, o modelo NRTL para determinar os coeficientes de atividade da fase liquida e o algorítimo de Levenberg-Marquartd para encontrar os ótimos locais dos parâmetros minimizando a função objetivo (FO) (Equação 5), onde os parâmetros $\mathrm{W}_{\mathrm{T}}$ e $\mathrm{Wy}$ seção definidos pelo usuário, sob as condições de variarem entre 0 e 1 e o somatório de $\mathrm{W}_{\mathrm{T}}$ e $\mathrm{Wy}$ ser 1 , estes parâmetros representam os pesos dados a cada desvio no cálculo da função objetivo e no presente trabalho adotou-se pesos de 0,5 para ambos .

$$
F O=\mathbf{W}_{\mathbf{T}} * \frac{100}{n} * \sum_{i=1}^{n}\left(\frac{T_{i}^{\text {calc }}-\tau_{i}^{\text {exp }}}{\tau_{i}^{\exp }}\right)+\mathbf{W}_{\mathbf{y}} * \frac{100}{n} * \sum_{i=1}^{n}\left(\frac{y_{1}{ }_{i}^{\text {calc }}-y_{1}{ }_{i}^{\text {exp }}}{y_{1}{ }_{i}^{\text {exp }}}\right)
$$

onde $\mathrm{W}_{\mathrm{T}}$ e $\mathrm{Wy}$ são os pesos atribuídos à temperatura e a composição em fase vapor, respectivamente. Para otimizar a entrada de dados e as etapas do algoritmo, foi desenvolvida uma interface gráfica. A otimização dos parâmetros ajustáveis foi feita pelo método de Levenberg-Marquardt. Os valores de pressão, temperatura e composição foram aproximados pelo método preditivo baseado na abordagem Gamma-Phi (NRTL - PR) no qual o modelo determina estas propriedades ajustando-as de acordo com o critério de equilíbrio que estipula que o somatório das frações molares em cada fase deve ser o mais próximo possível de 1,0.

\section{RESULTADOS E DISCUSSÕES}

Para verificar a consistência do modelo, um algoritmo preditivo já validado (CHECALC, 2015) foi aplicado para sistemas mais simples e seus resultados foram 
comparados com os fornecidos. Este teste foi realizado com o sistema agua-propanol para o qual variou-se a pressão de 0.005 a 0.5 bar obtendo-se uma boa correspondência com desvios inferiores a $0,3 \%$ inclusive nos pontos do azeótropo. As propriedades termodinâmicas dos componentes envolvidos na obtenção do biodiesel (DIPPR, 2000) são mostradas na Tabela 1.

Tabela 1 - Propriedades termodinâmicas dos componentes no biodiesel

\begin{tabular}{|c|c|c|c|c|c|c|c|}
\hline \multirow{2}{*}{ Substância } & \multirow{2}{*}{$\begin{array}{c}\text { Pc } \\
\text { (bar) }\end{array}$} & \multirow{2}{*}{$\begin{array}{l}\mathrm{Tc} \\
(\mathrm{K})\end{array}$} & \multirow{2}{*}{$\mathrm{W}$} & \multicolumn{3}{|c|}{$\mathrm{P}^{\mathrm{SAT}}$ (a) } & \multirow{2}{*}{$\begin{array}{c}\mathrm{Vl} \\
(\mathrm{L} / \mathrm{mol})\end{array}$} \\
\hline & & & & Ant a & Ant b & Ant c & \\
\hline metanol $^{\mathrm{b}}$ & 80,90 & 512,60 & 0,564 & 9,2025 & 1056,52 & $-85,9724$ & 0,0407 \\
\hline águab & 220,55 & 647,10 & 0,345 & 10,1620 & 1718,37 & $-39,9580$ & 0,0181 \\
\hline etanol $^{\mathrm{b}}$ & 61,48 & 513,90 & 0,645 & 11,0835 & 2085,87 & $-7,7520$ & 0,0587 \\
\hline glicerol $^{\mathrm{b}}$ & 66,80 & 726,00 & 0,934 & 8,7365 & 1356,47 & $-200,000$ & 0,0730 \\
\hline estearato de etila ${ }^{c}$ & 10,51 & 777,22 & 1,009 & 5,6797 & 1276,84 & 11,5600 & 0,3627 \\
\hline palmitato de etila ${ }^{c}$ & 11,80 & 759,02 & 0,931 & 5,9527 & 1602,59 & 78,5300 & 0,3311 \\
\hline oleato de etila ${ }^{c}$ & 10,49 & 776,88 & 0,995 & 5,2087 & 1187,11 & 20,9700 & 0,3573 \\
\hline laurato de etila ${ }^{c}$ & 15,35 & 715,99 & 0,771 & 3,8910 & 604,62 & 14,1967 & 0,2655 \\
\hline miristato de etila ${ }^{c}$ & 13,38 & 738,78 & 0,852 & 4,4224 & 802,26 & 13,6854 & 0,2982 \\
\hline linoleato de etila ${ }^{\mathrm{c}}$ & 10,48 & 776,54 & 0,981 & 8,0466 & 2969,27 & 161,3364 & 0,3525 \\
\hline
\end{tabular}

(a) Pressão de saturação: $P_{i}^{\text {sat }}=10^{\left(\text {Anta }-\frac{\text { Antb }}{t-A n t c)}\right)}$ : (b) $\mathrm{T}$ dada em Kelvin e $\mathrm{P}^{\text {sat }}$ resultante é dada em $\mathrm{kPa}$; (c) $\mathrm{T}$ dada em Celsius e $\mathrm{P}^{\text {sat }}$ resultante é dada em atm

A modelagem termodinâmica feita neste trabalho foi aplicada a sistemas binários formados por ésteres etílicos de ácidos graxos, etanol, glicerol, metanol e água. Os dados experimentais de ELV de alguns dos 13 sistemas binários foram obtidos da literatura científica e alguns são apresentados na Tabela 2 e os resultados se apresentam na Tabela 3.

Tabela 2 - Algumas características termodinâmicas dos dados experimentais de alguns sistemas binários estudados neste trabalho

\begin{tabular}{lccccc}
\hline \multicolumn{1}{c}{ Sistemas binários } & $\begin{array}{c}\Delta \mathrm{P} \\
(\mathrm{bar})\end{array}$ & $\begin{array}{c}\Delta \mathrm{T} \\
(\mathrm{K})\end{array}$ & $\Delta \mathrm{x}_{1}$ & $\Delta \mathrm{y}_{1}$ & Ref. \\
\hline etil palmitato + etil estearato & 0,0533 & $502,3-520,6$ & $0,0-1,0$ & $0,000-1,000$ & SILVA (2010) \\
etil palmitato + etil oleato & $0,0533-0,0933$ & $502,3-536,8$ & $0,0-1,0$ & $0,000-1,000$ & SILVA (2010) \\
etil palmitato + etil linoleato & 0,0933 & $514.9-537,7$ & $0,0-1,0$ & $0,000-1,000$ & SILVA (2010) \\
etil miristato + etil palmitato & $0,050-0,01500$ & $420,5-468,1$ & $0,0-1,0$ & $0,000-1,000$ & TANG et al, (2013) \\
etanol + etil estearato & 0,9199 & $350,9-418,9$ & $0,0-1,0$ & 1,000 & COELHO (2011) \\
etanol + etil palmitato & 1,0000 & $355,9-382,3$ & $0,0-1,0$ & $0.997-1,000$ & COELHO (2011_ \\
\hline
\end{tabular}

De um modo geral, os resultados obtidos pela modelagem foram bons, embora, alguns desvios tenham ocorrido a baixas pressões, com o aumento da pressão se obteve uma boa correlação entre os dados experimentais e calculados, por exemplo para o sistema etil miristato (1) + etil palmitato (2) avaliado a pressões de 0,005 bar, 0,01 bar e 0,015 bar obtiveram-se desvios de temperatura de 1,694, 1,258 e 0,680 respectivamente, a real expressão do significado destes desvios pode ser vista na Figura 1 onde se representa diagrama ELV referente ao sistema etil miristato (1) + etil palmitato (2) a 0.015 bar. Uma possível hipótese para esse desvio está no fato de não haver uma uniformidade na literatura com relação aos dados e propriedades críticas dos sistemas de ésteres etílicos fazendo com 
que os mesmos sejam mais sensíveis a desvios quando analisados sob condições menores que um bar.

Tabela 3 - Resultados obtidos da modelagem termodinâmica do ELV - abordagem Gamma-

Phi e método Bolha-T para sistemas binários

\begin{tabular}{lcccccc}
\hline \multicolumn{1}{c}{ Sistemas binários } & $\begin{array}{c}\mathrm{P} \\
(\mathrm{bar})\end{array}$ & $\begin{array}{c}\mathrm{g}_{12} \\
(\mathrm{cal} / \mathrm{mol} . \mathrm{K})\end{array}$ & $\begin{array}{c}\mathrm{g}_{12} \\
(\mathrm{cal} / \mathrm{mol} . \mathrm{K})\end{array}$ & $\alpha$ & $\begin{array}{c}\Delta \mathrm{T}^{\mathrm{a}} \\
(\%)\end{array}$ & $\begin{array}{c}\Delta \mathrm{y}_{1}^{\mathrm{b}} \\
(\%)\end{array}$ \\
\hline etil palmitato + etil estearato & 0,0533 & 595,60 & $-303,80$ & 0,3055 & 0,182 & 1,03 \\
etil palmitato + etil oleato & 0,0533 & 32,41 & $-21,00$ & 0,3046 & 0,162 & 1,67 \\
etil palmitato + etil linoleato & 0,0933 & 372,41 & $-405,57$ & 0,3145 & 0,341 & 2,82 \\
etil miristato + etil palmitato & 0,0150 & 48,86 & 48,88 & $-3,541$ & 0,680 & 2,93 \\
etanol + etil estearato & 0,9199 & 2659,04 & $-923,72$ & 0,2000 & 0,450 & 0,00 \\
etanol + etil palmitato & 1,0000 & 1848,20 & $-527,28$ & 0,2000 & 0,696 & 0,006 \\
\hline
\end{tabular}

$$
\Delta \mathbf{T}=\frac{100}{n} * \sum_{i=1}^{n}\left(\frac{T_{i}^{\text {calc }}-T_{i}^{\text {exp }}}{\tau_{i}^{\text {exp }}}\right), \text { (b) } \Delta \mathbf{y}_{\mathbf{1}}=\frac{100}{n} * \sum_{i=1}^{n}\left(\frac{y_{1}^{\text {calc }}-y_{i}{ }_{i}^{\text {exp }}}{y_{1}^{\text {exp }}}\right)
$$

Figura 1 - ELV do sistema: etil miristato (1) + etil palmitato (2) $(\mathrm{P}=0.015$ bar $)$

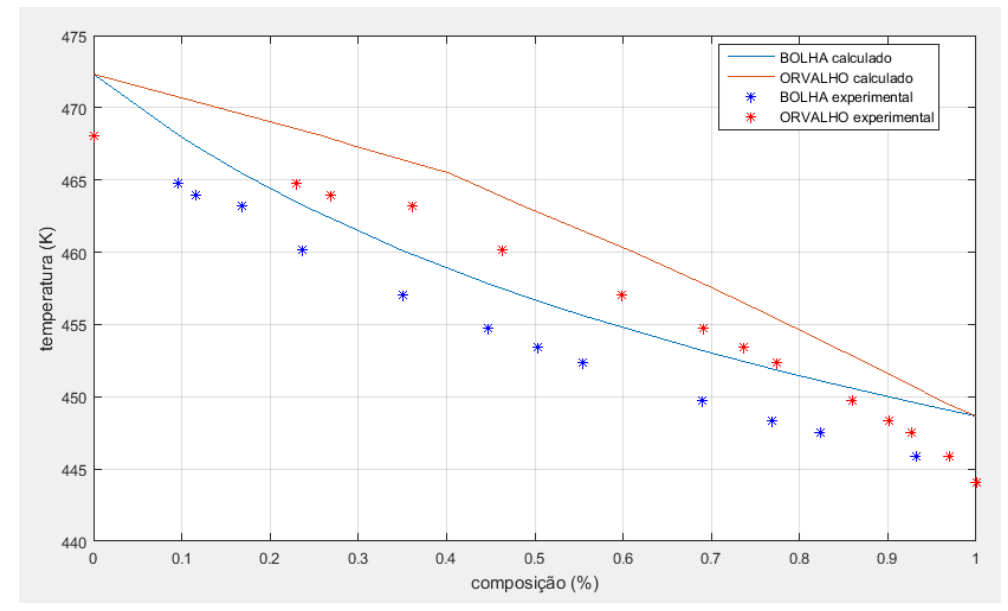

Outras possíveis hipóteses para a ocorrência destes desvios são, a dependência dos parâmetros NRTL da temperatura, fazendo com que por mais que se busquem parâmetros que melhor representem um conjunto de pontos, estes não sejam os parâmetros que melhor representam cada ponto individualmente. Os dados de propriedades críticas diferem muito de autor para autor gerando dúvida quanto a qual conjunto está mais próximo dos valores verdadeiros. Outros fatores que podem ser fontes de erro são as constantes de Antoine para o cálculo das pressões de saturação e os dados experimentais. Seria interessante realizar o teste de consistência termodinâmica desses dados experimentar utilizando a Eq. de Gibbs-Duhem.

\section{CONCLUSÕES.}

A grande dificuldade foi a coleta e a adaptação de dados referentes a propriedades, pois, além do fato de muitos autores não explicitarem as unidades de determinadas propriedades em seus trabalhos, não há uma uniformidade nas informações encontradas, de modo que as mesmas propriedades coletadas em artigos diferentes divergem mesmo nas mesmas condições, fato que se torna evidente quando se trabalha com moléculas de estruturas complexas. O modelo preditivo Gamma-Phi, NRTL-PR, é ideal para baixas pressões e foi 
aplicado para sistemas envolvendo componentes presentes no biodiesel com resultados razoáveis em termos dos desvios na temperatura e composição na fase vapor (Bolha T).

\section{REFERÊNCIAS}

CHECALC: BINARY VLE. Disponível em: <https://checalc.com/solved/binary_vle.html>. Acesso em: 22 abr. 2017

COELHO, R.A. Equilíbrio líquido-vapor de sistemas binários envolvendo ésteres etílicos do biodiesel (glicerol ou água) + etanol: Dados experimentais e modelagem termodinâmica. Dissertação (Mestrado). Universidade Federal do Paraná Curitiba, 2011.

DIPPR. Information and Data Evaluation Manager. Versão 1.2.0, 2000.

MENDES, D.B.; SERRA, J.C.; VALDÉS. Glicerina: uma abordagem sobre a produção e o tratamento. Revista Liberato, Novo Hamburgo, 2012.

PACHECO, E.M. Biodiesel. Brasília: Ministério da Educação, 2006. p. 6-12. Disponível em: <http://portal.mec.gov.br/setec/arquivos/pdf/cartilha_biodiesel.pdf >. Acesso em: 12 mar. 2017.

PENG, D.Y.; ROBINSON, D.B. A new two-constant equation of state. Ind. Eng. Chem. Fundam., v. 15, nº 1, p. 59-64, 1976.

RENON, H.; PRAUSNITZ, J.M. Local composition in thermodynamics excess functions for liquid mixtures. AIChE J., v. 14, p. 135-144, 1968

SILVA, L.Y.A. Determinação Experimental de Dados de Pressão de Vapor e de Equilíbrio Líquido-Vapor de Componentes do Biodiesel através da Calorimetria Exploratória Diferencial. 2010. 96 f. Tese (Doutorado) - Curso de Engenharia Química, Universidade Estadual de Campinas, Campinas, 2010.

SMITH, J.M.; VAN NESS, H. C.; ABBOTT, M. M. Introdução a termodinâmica da engenharia quimica. Rio de Janeiro: 7. ed., Editora LTC, 2005.

TANG, G; DING, H; HOU, J; XU, S. Isobaric vapor-liquid equilibrium for binary system of ethyl myristate + ethyl palmitate at 0.5, 1.0 and $1.5 \mathrm{kPa}$. Fluid Phase Equilib., v. 347, p. $8-14,2013$

TERRON, L.R. Termodinâmica Química Aplicada. Barueri, SP: Editora Manole, 2009.

\section{AGRADECIMENTOS}

L.P. Barreiros agradece à Escola de Engenharia de Lorena (EEL) da Universidade de São Paulo (USP) pela oportunidade de realizar a Iniciação Científica. P.F. Arce Castillo agradece à Fundação de Amparo à Pesquisa do Estado de São Paulo (FAPESP) através do processo 2015/05155-8 pelo auxílio financeiro à pesquisa. 